\title{
Vergleichende Untersuchungen über die Zusammensetzung und den Aufbau verschiedener Seidenarten.
}

I. Mitteilung.

Die Monoaminosäuren der aNew Chwang-Seides.1)

Von

Emil Abderhalden und Anguste Rilliet, Genf.

(Aus dem physiologischen Institute der tierärztlichen Hochschule, Berlin.)

(Der Redaktion zugegangen am 22. Dezember 1908.)

Die zu dieser Untersuchung verwendete Seide stammt aus China. Es war leider bis jetzt nicht möglich, genaue Daten über die Art der Seidenraupe und ihre Nahrung zu erhalten. Vermutlich lebt die Raupe von Eichenblättern. New ChwangSeide stellt ein bestimmtes Handelsprodukt dar. Wir hatten Gelegenheit, verschiedene Proben derselben Seidenart zu untersuchen. Die Resultate waren stets ganz ähnlich, so daß wir wohl annehmen dürfen, daß uns ein ganz bestimmtes Gewebe vorgelegen hat. Wir verwendeten das rohe Gespinnst. Zunächst wurde die braun gefärbte Seide mechanisch durch Zerzupfen und Ausschütteln von Staub und sonstigen Beimengnngen sorgfältig gereinigt. Dann wurde das Gewicht der Seide festgestellt, und nunmehr der Seidenleim durch Auskochen mit Wasser in einem Porzellangefäß im Autoklaven entfernt. Das Wasser wurde so lange erneuert, bis beim Eindampfen des Auszuges kein erheblicher Rückstand mehr blieb. Etwas ging immer noch in Lösung. Es scheint, daß das Seidenfibroin trotz aller Vorsichtsmaßregeln - Anwendung von destilliertem Wasser

1) Vgl. hierzu: Emil A bderhalden, Vergleichende Untersuchungen über die Zusammensetzung und den Aufbau verschiedener Seidenarten, Diese Zeitschrift, Dieser Band, S. 334, 1909. 
und Benutzung eines Porzellangefäßes - beirn Erhitzen mit Wasser unter Druck angegriffen wird. Die Menge des Seidenleims wurde durch Eindampfen aller wässerigen Auszüge bestimmt. Das Seidenfibroin wurde zur Bestimmung des Tyrosins in der gewohnten Weise mit 25\% iger Schwefelsäure hydrolysiert und zur Gewinnung der übrigen Aminosäuren mit rauchender Salzsäure gekocht. Beim Kochen mit den genannten Säuren machten wir die Beobachtung, daß die Hydrolyse langsamer erfolgt, als dies z. B. bei dem Mailänder Fibroin der Fall ist. Nach sechsstündigem Kochen des Seidenfibroins mit rauchender Salzsäure war ein erheblicher Teil noch ungelöst. Er wurde abfiltriert und nochmals mit rauchender Salzsäure gekocht. Bei Anwendung von $220 \mathrm{~g}$ rohen Seidenfibroins verblieb schließlich ein Rückstand von $35,5 \mathrm{~g}$. Bei einer zweiten Hydrolyse verblieben von den verwendeten $200 \mathrm{~g} \mathrm{28,0} \mathrm{g}$. Auch bei der $\mathrm{Hy}-$ drolyse mit 25\% iger Schwefelsäure blieb ein großer Teil ungelöst. Auch hier wurde dieser Rückstand nochmals mit Schwefelsäure gekocht. Es verblieb nach der Wiederholung des jedesmal 18 stündigen Erhitzens mit der genannten Säure ein Kückstand von 24,6 $\mathrm{g}$ bei Anwendung von $83 \mathrm{~g}$ Fibroin. In beiden Fällen war dieser Rückstand tief schwarz gefärbt. Mit seiner Untersuchung sind wir noch beschäftigt.

Auf die Beschreibung des Ganges der Untersuchung können wir um so eher verzichten, als er an dieser Stelle wiederholt geschildert worden ist. Es seien nur die Resultate angeführt.

$120 \mathrm{~g}$ Seide verloren nach viermaligem, je dreistündigem Auskochen mit je $3 \mathrm{l}$ Wasser $24 \mathrm{~g}$ Leim $=20 \%$.

$210 \mathrm{~g}$ Seide verloren nach viermaligem, je vierstündigem Auskochen mit je $4 \mathrm{l}$ Wasser $39,8 \mathrm{~g}$ Leim $=18,95 \%$.

$200 \mathrm{~g}$ Seide verloren nach viermaligem, je fünf Stunden dauerndem Auskochen mit je $4 \mathrm{l}$ Wasser 40,0 g Leim $=20 \%$.

Die verwendete Seide verlor beim Trocknen bei $120^{\circ}$ durchschnittlich $10 \%$ an Gewicht. Sie enthielt ferner durchschnittlich $5 \%$ Asche. Von Aschenbestandteilen konnten Eisen, Calcium, Phosphorsäure und Salzsäure qualitativ nachgewiesen werden. Die getrocknete degommierte Seide enthielt noch $2 \%$ Asche. Die folgenden Resultate beziehen sich alle auf das bei 
Über die Zusammensetzung verschiedener Seidenarten. I. 339

$120^{\circ}$ getrocknete Seidenfibroin und zwar nach Abzug der Asche und des nicht hydrolysierbaren Anteils.

Zur Bestimmung des Tyrosins verwendeten wir 57,0 g Fibroin. Erhalten wurden 7,2 g rohes Tyrosin. Nach dem Umkrystallisieren aus heißem Wasser betrug die Ausbeute 5,6 g $=\mathbf{9}, 83 \%$.

Zum Nachweis der übrigen Aminosäuren wurden $205 \mathrm{~g}$ Fibroin (nach Abzug der Asche und des nicht hydrolysierbaren Anteils) verwendet. Die Veresterung wurde in der gewohnten Weise durchgeführt. Es konnten 65,2 g Glykokollesterchlorhydrat vom Schmelzpunkt $144^{\circ}$ (korr.) direkt abgeschieden werden.

Die Ester wurden aus den Esterchlorhydraten mit Natronlauge und Kaliumcarbonat in Freiheit gesetzt. Die Destillation der Aminosäureester erfolgte in drei Fraktionen. Die erste wurde von $0-100^{\circ}$ des Wasserbades bei $10 \mathrm{~mm}$ Druck aufgefangen, die zweite bis $100^{\circ}$ des Wasserbades bei $0,2 \mathrm{~mm}$ Druck und die letzte von $100-180^{\circ}$ des Ölbades bei $0,3 \mathrm{~mm}$ Druck.

Die beiden ersten Fraktionen wurden mit Wasser, die dritte mit Baryt verseift, nachdem in der gewohnten Weise aus letzterer der Phenylalaninester durch Ausäthern entfernt worden war.

Die verseiften beiden ersten Fraktionen wurden zunächst unter vermindertem Druck bei $35^{\circ}$ des Wasserbades zur Trockene verdampft. Der Rückstand wurde zur Entfernung des Prolins mit absolutem Alkohol ausgekocht und dann der in Alkohol unlösliche Teil fraktioniert krystallisiert. An Prolin erhielten wir $3,8 \mathrm{~g}=1,85 \%$. Identifiziert wurde es durch die Analyse seines Kupfersalzes.

$0,1275 \mathrm{~g}$ racemisches, lufttrockenes Prolinkupfer verloren bei $140^{\circ}$ $0,0138 \mathrm{~g}$ Wasser.

Berechnet für $\mathrm{C}_{10} \mathrm{H}_{16} \mathrm{O}_{4} \mathrm{~N}_{2} \mathrm{Cu}+2 \mathrm{H}_{2} \mathrm{O}$ : Gefunden:

$$
10,99 \% \mathrm{H}_{2} \mathrm{O} \text {. } 10,82 \% \mathrm{H}_{2} \mathrm{O} \text {. }
$$

$0,2632 \mathrm{~g}$ Substanz gaben $0,0631 \mathrm{~g} \mathrm{CuO}=0,0504 \mathrm{~g} \mathrm{Cu}$.

Berechnet für $\mathrm{C}_{10} \mathrm{H}_{16} \mathrm{O}_{4} \mathrm{~N}_{2} \mathrm{Cu}+2 \mathrm{H}_{2} \mathrm{O}$ : Gefunden: $19,41 \% \mathrm{Cu}$.

$19,14 \% \mathrm{Cu}$.

Die erste Fraktion enthielt $42 \mathrm{~g}$ Alanin und 5,5 g Glykokoll. Aus der zweiten Fraktion gewannen wir $7 \mathrm{~g}$ Alanin und 3,2 $\mathrm{g}$ Leucin. Wahrscheinlich ist auch Valin in geringen 
Mengen vorhanden. Es konnte jedoch nicht genügend identifiziert werden. Das isolierte d-Alanin zeigte $[\alpha]_{20^{\circ}}^{\mathrm{D}}=+9,8^{\circ}$ in der berechneten Menge Salzsäure gelöst.

$0,1070 \mathrm{~g}$ Alaninkupfer gaben $0,0355 \mathrm{~g} \mathrm{CuO}=0,0283 \mathrm{~g} \mathrm{Cu}$.

Berechnet für $\left(\mathrm{C}_{3} \mathrm{H}_{6} \mathrm{NO}_{2}\right)_{2} \mathrm{Cu}$ :

Gefunden :

$26,41 \% \mathrm{Cu}$.

$26,51 \% \mathrm{Cu}$.

Vom Leucin wurde gleichfalls das Kupfersalz untersucht: $0,1830 \mathrm{~g}$ Kupfersalz gaben $0,0460 \mathrm{~g} \mathrm{CuO}=0,0367 \mathrm{~g} \mathrm{Cu}$.

Berechnet für $\left(\mathrm{C}_{6} \mathrm{H}_{12} \mathrm{NO}_{2}\right)_{2} \mathrm{Cu}$ : $19,6 \% \mathrm{Cu}$. Gefunden : $20,0^{\circ} \% \mathrm{Cu}$.

Aus der dritten Fraktion gewannen wir 2,5 g Phenylalanin, 3,5 g Glutaminsäure, 5,8 g Asparaginsäure und $2,0 \mathrm{~g}$ Serin.

\section{Asparaginsäure:}

- $0,2080 \mathrm{~g}$ Substanz gaben $0,2760 \mathrm{~g} \mathrm{CO}_{2}$ und 0,1028 $\mathrm{g} \mathrm{H}_{2} \mathrm{O}$.

Berechnet für $\mathrm{C}_{4} \mathrm{H}_{7} \mathrm{NO}_{4}$ :

Gefunden:

$36,09 \% \mathrm{C}$ und $5,26 \% \mathrm{H}$.

$36,19 \% \mathrm{C}$ und $5,53 \% \mathrm{H}$.

Phenylalanin:

0,1480 g Substanz gaben $0,3532 \mathrm{~g} \mathrm{CO}_{2}$ und $0,0899 \mathrm{~g} \mathrm{H}_{2} \mathrm{O}$.

Berechnet für $\mathrm{C}_{9} \mathrm{H}_{11} \mathrm{NO}_{2}$ :

Gefunden :

$65,45 \% \mathrm{C}$ und $6,66 \% \mathrm{H}$. $65,09 \% \mathrm{C}$ und $6,79 \% \mathrm{H}$.

Serin:

0,1510 g Substanz gaben 0,1886 g $\mathrm{CO}_{\mathrm{z}}$ und $0,0950 \mathrm{~g} \mathrm{H}_{2} \mathrm{O}$.

Berechnet für $\mathrm{G}_{3} \mathrm{H}_{7} \mathrm{NO}_{3}$ :

Gefunden:

$34,28 \% \mathrm{C}$ und $6,66^{\circ} \% \mathrm{H}$.

$34,06{ }^{\circ}$ \% $\mathrm{C}$ und $6,99 \% \mathrm{H}$.

Die Ausbeute an Serin ist unzweifelhaft zu gering, denn aus dem Destillationsrückstand ließ sich noch Serinanhydrid isolieren. Seine Menge betrug 0,75 g. Es war jedoch nicht ganz rein.

Auf $100 \mathrm{~g}$ reines Seidenfibroin berechnet, ergeben sich folgende Ausbeuten an Aminosäuren:

$\begin{array}{lr}\text { Glykokoll } & 19,7 \mathrm{~g} \\ \text { Alanin } & 23,8 \text {, } \\ \text { Leucin } & 1,6 \text {, } \\ \text { Serin } & 1,0 \text { ” } \\ \text { Asparaginsäure } & 2,9 \text { " } \\ \text { Glutaminsäure } & 1,7 \text { " } \\ \text { Phenylalanin } & 1,2 \text { " } \\ \text { Tyrosin } & 9,8 \text { " } \\ \text { Prolin } & 1,85 \text { " }\end{array}$

